\title{
Large Right Atrial Myxoma Masquerading as Malignancy
}

\author{
Bharath $\mathrm{V}^{1, \odot} \quad$ Milind Padmakar Hote ${ }^{1}$
}

\begin{abstract}
Address for correspondence Bharath $\mathrm{V}$, Department of Cardiothoracic and Vascular Surgery, All India Institute of Medical Sciences, New Delhi, 110029, India (e-mail:drbharathv@gmail.com).
\end{abstract}
Abstract
Keywords
- right atrial mass
- myxoma
- benign tumor
- malignancy

\begin{abstract}
A 57-year-old female presented to emergency with features of right heart failure. On evaluation, she was found to have a large mass occupying right atrium (RA) completely and protruding into right ventricle through tricuspid valve. Intraoperatively, mass was seen arising from RA free wall with stony hard consistency. Histopathology revealed it to be myxoma. We present this case for the rare presentation of myxoma masquerading as malignancy.
\end{abstract}

\section{Introduction}

Myxoma is the most common benign tumor of heart. ${ }^{1}$ Usually, myxomas are soft, lobulated, and friable masses. ${ }^{2}$ Its seen commonly in left atrium (LA), right atrium (RA), and less frequently in ventricles. Due to its friable nature, it can easily embolize distally. It is usually diagnosed when person develops heart failure symptoms or embolic phenomenon. It rarely presents as a solid mass with involvement of ventricle along with atrium.

\section{Case Report}

A 57-year-old woman presented to emergency with shortness of breath and generalized body swelling for 2 months. Echocardiography revealed solid mass in RA and its protrusion through tricuspid valve into right ventricle (RV). There was severe tricuspid regurgitation due to the mass.

She underwent contrast-enhanced CT angiography for evaluation of coronaries and to rule out distal embolization

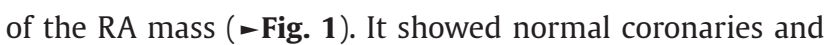
a solid mass arising from RA free wall. Both the vena cavae were free of mass.
The patient was planned for emergency mass excision. Once intubated and on mechanical ventilation, with arterial and central venous lines secured, she was placed in supine position, painted and draped.

Midline sternotomy was performed and pericardial opened vertically. An enlarged RA was visualized. Since the mass was not involving superior and inferior vena cava (SVC and IVC), central cannulation was performed, and cardiopulmonary bypass established. RA was opened after cross-clamping aorta and pulmonary artery and arresting the heart in diastole with cold blood cardioplegia.

Just as the RA was incised, the mass was seen completely occupying it and bulging out ( - Figs. 2,3 ).

The mass was gently delivered from RV into RA and dissected off its attachment from the lateral wall of RA. RV was inspected for embolic mass and thoroughly washed with cold saline. Tricuspid patency was confirmed with saline test and transesophageal echocardiography.

RA was closed and patient weaned off bypass. Routine decannulation and closure followed. - Fig. 4 shows measurements of the mass.

Histopathological analysis revealed the mass to be myxoma with hemorrhagic areas within. published online August 5, 2021
DOI https://doi.org/

$10.1055 / \mathrm{s}-0041-1732843$ ISSN 2457-0206
(C) 2021. Official Publication of The Simulation Society (TSS), accredited by International Society of Cardiovascular Ultrasound (ISCU).

This is an open access article published by Thieme under the terms of the Creative Commons Attribution-NonDerivative-NonCommercial-License, permitting copying and reproduction so long as the original work is given appropriate credit. Contents may not be used for commercial purposes, or adapted, remixed, transformed or built upon. (https://creativecommons.org/licenses/by-nc-nd/4.0/).

Thieme Medical and Scientific Publishers Pvt. Ltd. A-12, 2nd Floor, Sector 2, Noida-201301 UP, India 


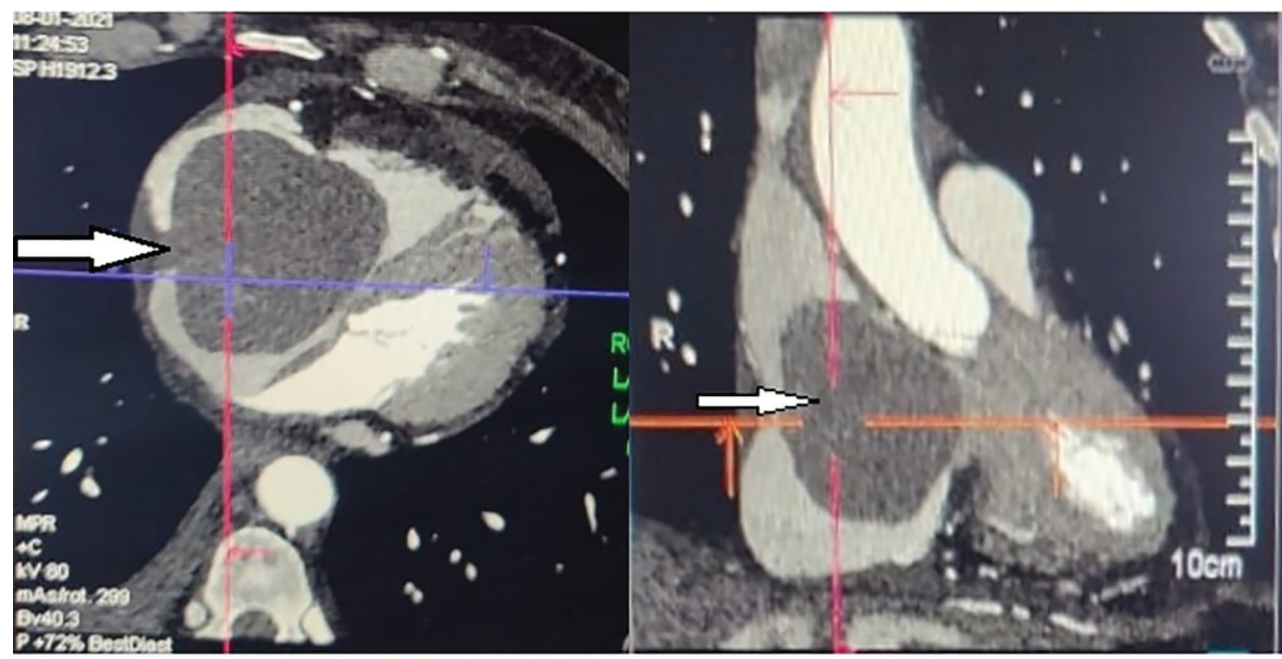

Fig. 1 Contrast-enhanced CT image showing mass occupying whole of right atrium (RA) (white arrow).

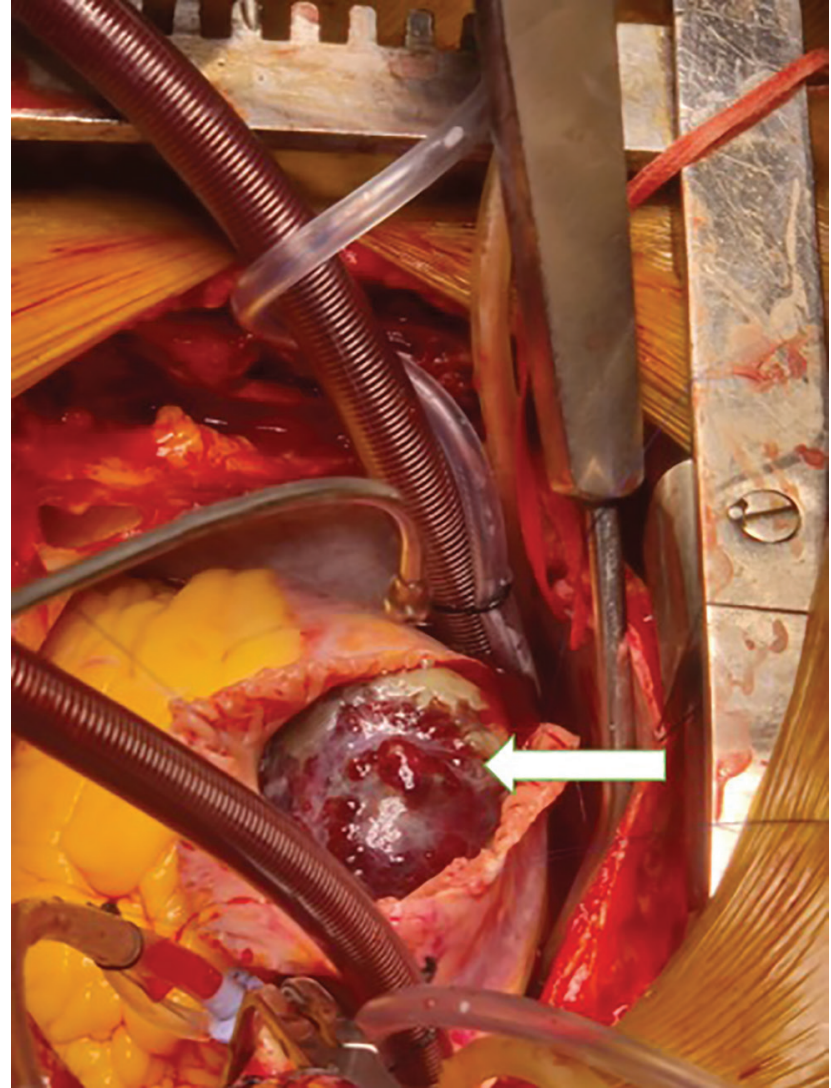

Fig. 2 Visualization of mass on opening right atrium (RA) (white arrow).

\section{Discussion}

Myxoma usually presents as a globular mass in atrium and rarely presents in ventricle. It has a globular appearance on echocardiography and CT. It rarely presents as a solid structure.

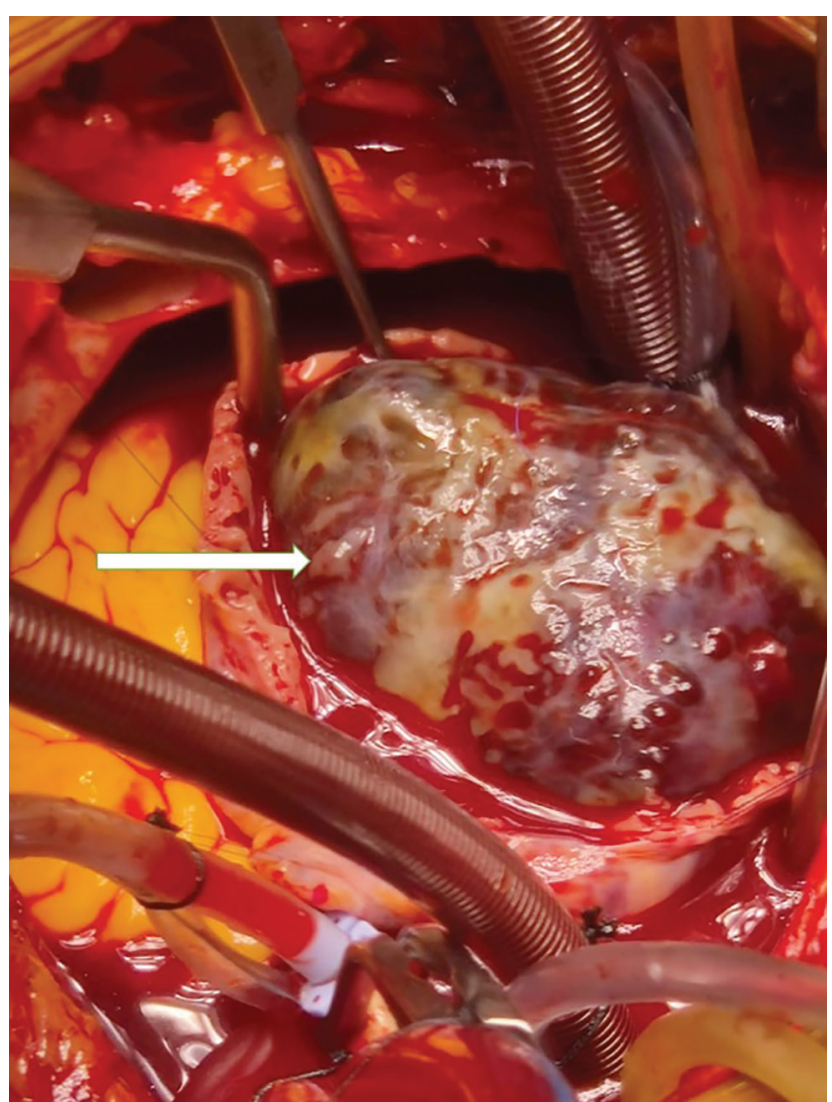

Fig. 3 Mass shown occupying whole of right atrium (RA) (white arrow).

Intraoperative appearance of the myxoma is also classical, like a bunch of grapes with a bluish hue. It is soft in consistency and easily friable. Management of myxoma involves excision in toto, with care taken to prevent embolization. ${ }^{3}$

Patients are followed-up for recurrence with transthoracic echocardiography. 


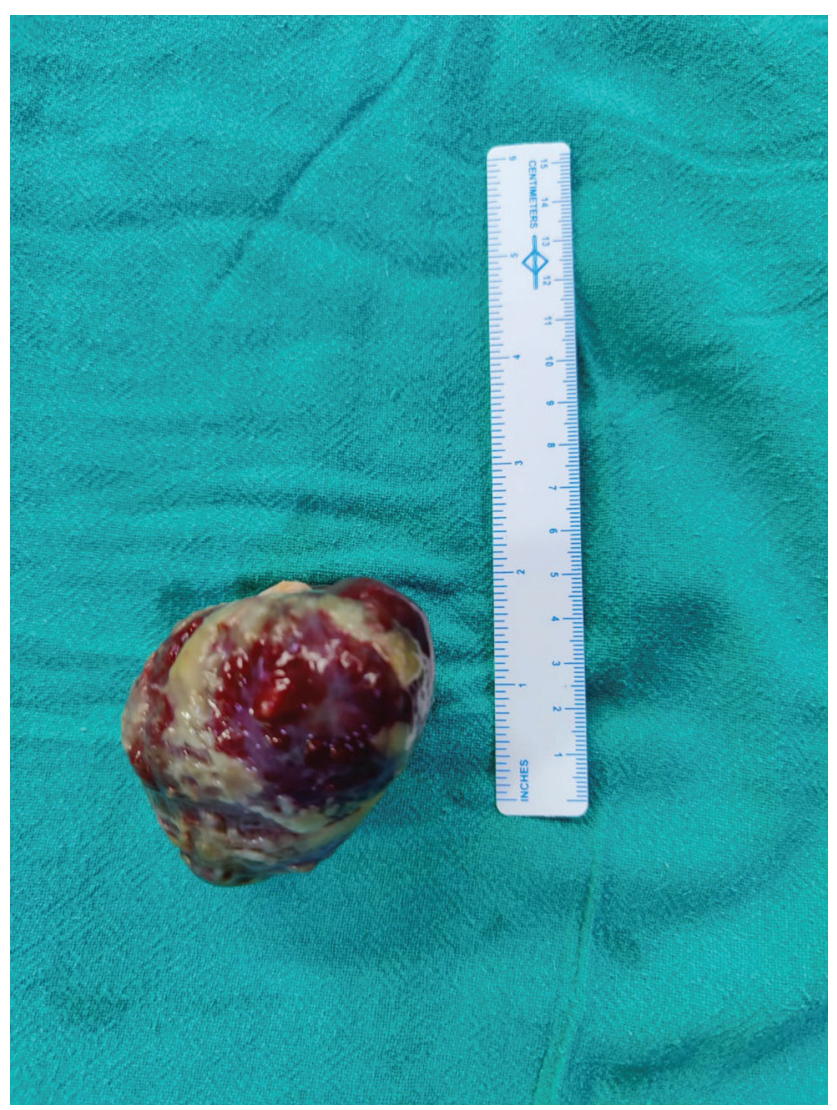

\section{Conclusion}

Myxomas are the most common benign tumors of the heart with characteristic features. They rarely present as a solid large mass. In such situations, it may masquerade as a malignancy.

\section{Funding}

None.

\section{Ethics Approval}

N/A.

\section{Consent for publication}

Taken.

\section{Conflicts of interest/Competing interest}

None declared.

\section{References}

1 Reynen K. Cardiac myxomas. N Engl J Med 1995;333(24):1610-1617

2 Xie X, Bai J, Li X. Right atrial myxoma. J Card Surg 2018;33(7):388-390

3 Choi J, de Costa A, Sabetai MM. Surgical management of a giant right atrial myxoma. J Surg Case Rep 2018;2018(10):rjy288

Fig. 4 Measurements. 